\title{
Pulsed Laser Powered Homogeneous Pyrolysis: A Computational Analysis
}

\author{
MARK T. SWIHART and ROBERT W. CARR \\ Department of Chemical Engineering and Materials Science, University of \\ Minnesota, 421 Washington Ave. S.E. Minneapolis, Minnesota 55455-0132
}

\begin{abstract}
The use of the pulsed laser powered homogeneous pyrolysis technique for measuring unimolecular decomposition rate constants under unambiguously homogeneous conditions is investigated by numerical simulation of the experiment. The coupled partial differential equations which govern the gas dynamics and chemical kinetics are solved numerically and the results analyzed. Conditions under which rate constants can be extracted from the experimental data using a simplified analysis are determined. The effects of five sources of error in the simplified analysis are computed. A correlation is presented which may be used to correct for overestimation of the rate constant which is inherent in the simple analysis. Conditions under which the other four sources of error become negligible are presented. Overall, it is expected that this technique will be capable of routinely measuring rate constants within a factor of 2 , and will do much better when a high power laser with a uniform beam profile is used and/or a well characterized thermal monitor molecule is available which decomposes with kinetic parameters close to that of the reactant being investigated. (C)1994 John Wiley \& Sons, Inc.
\end{abstract}

\section{Introduction}

Pulsed laser powered homogeneous pyrolysis (LPHP) is a technique which may be used to measure unimolecular decomposition kinetics at temperatures of 600- $1500 \mathrm{~K}$ with reaction times on the order of $10 \mu \mathrm{s}$ per pulse. This method has been used to measure bond dissociation energies and unimolecular decomposition rate constants for a variety of compounds [1-5]. A review of LPHP was recently given by Russell [6].

In a typical pulsed LPHP experiment, a pulsed $\mathrm{CO}_{2}$ laser is used to rapidly heat a gas mixture consisting of the reactant, a bath gas, and a photosensitizer such as $\mathrm{SiF}_{4}$ or $\mathrm{SF}_{6}$, to the desired reaction temperature. If the heated gas does not completely fill the reactor, it then expands and cools in a few microseconds, quenching the reaction. In this configuration, the laser heats only a column of gas near the center of the reactor, while the reactor walls and most of the reactor volume remain near room temperature. After many pulses, the final gas composition is measured. If the reaction temperature and reaction time are known, rate constants may then be calculated from the final composition. More complete descriptions of the technique and variations on it may be found in references [1-8].

The primary advantage of the LPHP technique is that the reactor walls are cold, so that all reaction which takes place is guaranteed to be homogeneous. This technique also allows rate measurements over a wide temperature range and with short reaction times which help to minimize the importance of secondary reactions. Also, the equipment required for the experiment is reasonably simple and inexpensive. The primary difficulties with this technique involve characterization of the reaction

International Journal of Chemical Kinetics, Vol. 26, 779-799 (1994)

(C) 1994 John Wiley \& Sons, Inc.

CCC 0538 8066/94/080779-21 
temperature and the reaction time. These may be measured or inferred by several methods including chemical thermometry [1-8] and time dependent thermal lensing $[8,9]$.

The analysis presented in this article has been developed with an eye toward the determination of unimolecular decomposition rate constants for organometallic compounds used in the manufacture of thin films by chemical vapor deposition. The pulsed LPHP technique is well-suited to the study of these compounds, since they are typically surface sensitive and thermally decompose at temperatures attainable with LPHP. Common assumptions made in the analysis of LPHP experiments are tested computationally here in order to assess the reliability of kinetic data and the prospects for using LPHP as a routine method for kinetics investigations. It is assumed throughout this analysis that the reaction whose rate is being measured is a simple unimolecular decomposition which produces unreactive products. Complications resulting from secondary reactions or unknown chemistry will be not be addressed.

\section{An Elementary Analysis}

The simplest analysis of the processes which occur in the reactor after a laser pulse is shown schematically in Figure 1. The geometry of the system is shown in Figure 1(a). The laser pulse heats a cylinder of gas which is taken to be concentric with the reactor volume. It is assumed that the laser heats the gas instantaneously, so that initially (at $t=0$ ) the gas is hot at positions inside the beam (region 1 in Fig. $1(\mathrm{~b})$ ) and cool at positions outside the beam (region 5). After the laser pulse, a weak shock wave, shown as a heavy line, travels outward from the beam radius $\left(\boldsymbol{r}=\boldsymbol{R}_{\boldsymbol{b}}\right)$, heating and compressing the gas slightly (region 4). Meanwhile, an expansion or rarefaction wave travels inward from the beam radius to the center of the heated cylinder. The head and tail of this expansion wave are shown as solid lines in Figure l(b). The head of the expansion wave travels at the speed of sound $\boldsymbol{a}=(\gamma R T / M)^{0.5}$, where $\boldsymbol{T}$ is the temperature ahead of the wave (region 1). Between the head and tail of the expansion wave (region 2) the temperature drops substantially, so that behind the expansion wave (region 3) the temperature may be as much as 200-300 K cooler than in region 1 . The boundary between the initially hot and cold regions, shown as a dotted line, moves outward as the gas expands and cools. When the waves reach the center line or the reactor wall, they are reflected, so that the cooling and heating processes are repeated.

In order to determine rate constants from measured concentrations, we may apply a simple analysis based on the wave propagation described above. In this analysis, all reaction is assumed to occur in front of the expansion wave (in region 1). Then, the final concentration of reactant at any point is given by $\mathrm{C}=\mathrm{Co} \exp (-\mathrm{kt})$, where $\boldsymbol{k}$ is the first-order rate constant for the reaction and $t$ is the time for the head of the expansion wave to reach that point. Since the head of the expansion wave travels at the speed of sound, $\boldsymbol{a}$, we have $\mathrm{t}=\left(R_{b}-\boldsymbol{r}\right) / \boldsymbol{a}$. If we define a dimensionless rate constant $\kappa=k R_{b} / a$ and integrate over the beam radius we get

$$
\left.\mathrm{C}=2 \mathrm{C}_{0}(\kappa-1+\exp (-\kappa)) / \kappa\right)
$$

for the average concentration inside the beam radius. Expanding the exponential in a Taylor series gives

$$
\mathrm{C} / \mathrm{C}_{0}=1-\kappa / 3+\kappa^{2} / 12-\kappa^{3} / 60+\text { higher order terms }
$$


(a)

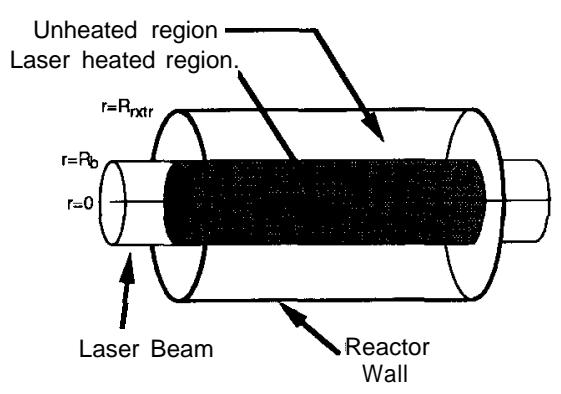

(b)

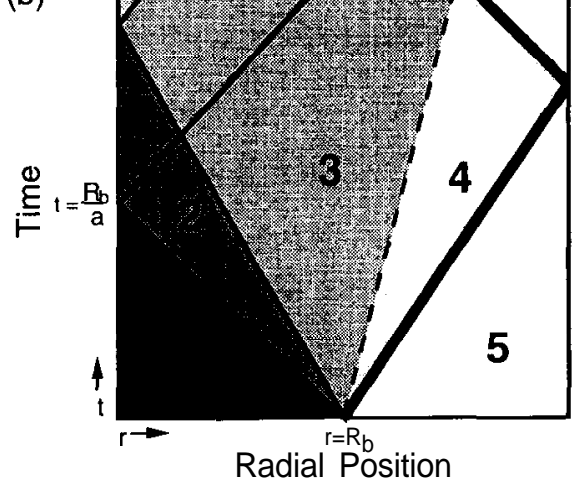

Figure 1. (a) Geometry of reactor. The laser beam and reactor are assumed cylindrically symmetric and concentric with radii $R_{b}$ and $R_{r x t r}$, respectively. The laser instantaneously heats a cylinder of gas of radius $R_{b}$ to a uniform temperature. (b) Simplified picture of shock and rarefaction wave propagation. The weak shock wave, shown as a heavy line, originates at $r=R_{b}$ and propagates outward, heating the gas behind it slightly. The expansion wave, the head and tail of which are shown as solid lines, originates at $r=R_{b}$ and propagates inward, cooling the gas behind it. The boundary between the initially hot and cold regions, shown as a dashed line, moves outward from $r=R b$. Regions of higher temperature are indicated by darker shading.

If the per-pulse conversion is small (less than about 1\%), then the quadratic and higher terms may be neglected, so we have the result that

$$
\text { conversion per pulse }=1-\mathrm{C} / \mathrm{Co}=\kappa / 3
$$

Thus, rate constants can be easily computed from the fractional conversion of the reactant after a number of pulses. The above expression gives the conversion of reactant in the irradiated region, which is easily related to the average conversion over the entire reactor, since no reaction occurs outside this region. The above analysis will be accurate when the temperature profile is initially uniform, the laser heating is instantaneous, and the expansion wave cooling is sufficient to quench all reaction behind the head of the expansion wave. Temperature uniformity requires that the portion of the laser beam used has an ideal "top-hat" profile, so that there is a uniform (high) temperature inside the beam, and a uniform (low) temperature outside the beam.

In this article, computational gas dynamics are applied to the pulsed LPHP situation in order to see when and how well the above analysis applies, and to quantify several sources of error in the LPHP experiment. While there has been some mention of computational gas dynamics in the LPHP literature [3,5,7], we have been unable to find full presentation of the results of a careful analysis. The sources of error investigated here include: (1) Overestimate of the rate constant due to the fact that some reaction inevitably occurs behind the head of the expansion wave (outside region 1); (2) The axially decreasing initial temperature distribution resulting from absorption of laser energy as the beam passes through the reactor; (3) The radially nonuniform temperature distributions that would result from a nonuniform laser energy profile; (4) The noninstantaneous establishment of the initial temperature distribution; and (5) Variations in the temperature from pulse-to-pulse due to variations in laser pulse energy. Quantitative estimates of errors in measured 
rate constants resulting from the above effects are presented, along with criteria for minimizing the effects and an estimate of the overall accuracy with which rate constants may be measured by this technique.

\section{Governing Equations and Their Solution}

The equations which govern the gas dynamics and reactant concentration in the LPHP experiment are differential mass, momentum and energy balances on the gas mixture in the reactor, and a species balance on the reactant. These were written and solved in terms of Eulerian position coordinates fixed in space, as opposed to Lagrangian coordinates which move with the fluid elements. The Lagrangian approach has been used in some previous calculations of LPHP gas dynamics [7], but the Eulerian point of view was more convenient for this particular analysis. The equations, as presented below, may be found in standard references [10]. The mass balance is the ordinary continuity equation with variable density.

$$
\frac{d \rho}{d t}+\nabla \cdot(\rho \underline{v})=0
$$

The momentum balance includes pressure forces and inertial forces, but no viscous forces.

$$
\frac{d(\rho \underline{v})}{d t}+\nabla \cdot(\rho \underline{v v})+\nabla p=0
$$

Likewise, the energy balance neglects thermal conduction and viscous dissipation of energy.

$$
\frac{d(\rho E)}{d t}+\nabla \cdot((\rho E+p) \underline{v})=S_{E}(r, z, t)
$$

A source term, $S_{E}(r, z, t)$, is included in the energy equation and is nonzero when energy is being deposited into the gas by the laser. Heat generation (consumption) due to reaction, however, is neglected. The species balance includes disappearance of a single reactant by a single first-order reaction with rate constant $k=A \exp \left(-E_{a} / R T\right)$ and neglects diffusion of the reactant.

$$
\frac{d(\rho w)}{d t}+\nabla \cdot(\rho w \underline{v})=-k p w
$$

The equation of state is a combination of the ideal gas law with the assumption of a constant heat capacity.

$$
p=\rho(\gamma-1)\left(E-\frac{|\underline{v}|^{2}}{2}\right)=\frac{\rho R T}{M}
$$

Temperature may be obtained via the ideal gas law, as shown in the second version of the equation of state. It also appears implicitly in the species balance through the Arrhenius temperature dependence of the rate constant. The equations were made dimensionless by appropriate scaling, and it is the dimensionless equations that were used in all computations presented here. Table I shows the dimensionless form of the equations in both their general form and the form they take for the l-dimensional, cylindrically symmetric situation considered in most of the calculations which follow. Dimensionless variables in the equations are defined as: 
TABLE I. Governing equations in dimensionless form.

\begin{tabular}{|c|c|c|}
\hline & General Form & $\begin{array}{l}\text { One-Dimensional } \\
\text { Cylindrical Geometry }\end{array}$ \\
\hline $\begin{array}{l}\text { Mass } \\
\text { Balance }\end{array}$ & $\frac{d \varphi}{d \tau}+\nabla \cdot(\varphi \underline{v})=0$ & $\frac{d \varphi}{d \tau}+\frac{1}{\eta} \frac{d}{d \eta}\left(\eta \varphi v_{r}\right)=0$ \\
\hline $\begin{array}{l}\text { Momentum } \\
\text { Balance }\end{array}$ & $\frac{d(\underline{\varphi} \underline{v})}{d \tau}+\mathrm{v}(\varphi \underline{v \underline{v}})+\frac{1}{\gamma} \nabla \pi=0$ & $\frac{d\left(\varphi v_{r}\right)}{d \tau}+\frac{1}{\eta} \frac{d}{d \eta}\left(\eta \varphi v_{r}^{2}\right)+\frac{1}{\gamma} \frac{d \pi}{d \eta}=0$ \\
\hline $\begin{array}{l}\text { Energy } \\
\text { Balance }\end{array}$ & $\frac{d(\varphi \xi)}{d \tau}+\nabla((\varphi \xi+\pi) \underline{u})=S_{\xi}(\eta, \zeta, \tau)$ & $\frac{d(\varphi \xi)}{d \tau}+\frac{1}{\eta} \frac{d}{d \eta}\left(\eta(\varphi \xi+\pi) v_{r}\right)=S_{E}(\eta, \tau)$ \\
\hline $\begin{array}{l}\text { Species } \\
\text { Balance }\end{array}$ & $\frac{d(\varphi \omega)}{d \tau}+\mathrm{V}(\varphi \omega \underline{v})=-\kappa \varphi \omega$ & $\frac{d(\varphi \omega)}{d \tau}+\frac{1}{\eta} \frac{d}{d \eta}\left(\eta \varphi \omega v_{r}\right)=-\kappa \varphi \omega$ \\
\hline $\begin{array}{l}\text { Equation } \\
\text { of State }\end{array}$ & $\pi=\varphi(\gamma-1)\left(\xi-\gamma \frac{|\underline{y}|^{2}}{2}\right)=\varphi \Theta$ & $\pi=\varphi(\gamma-1)\left(\xi-\gamma \frac{\underline{v}_{r}^{2}}{2}\right)=\varphi \Theta$ \\
\hline
\end{tabular}

$$
\begin{gathered}
\eta=r / R_{b} ; \quad \zeta=z / R_{b} ; \quad \tau=t a / R_{b} ; \quad \varphi=\rho / \rho_{0} ; \quad \underline{v}=\underline{v} / a \\
v_{r}=v_{r} / a ; \quad \Theta=T / T_{0} ; \quad \pi=p / p_{0} ; \quad \xi=E \rho_{0} / p_{0} \\
\omega=w / w_{0} ; \quad \gamma=\mathrm{C}_{p} / \mathrm{C}_{v} ; \quad \alpha=A R_{b} / a \\
\xi_{a}=E_{a} / R T_{0} ; \quad \kappa=k R_{b} / a=\alpha \exp \left(-\xi_{a} / \Theta\right) ;
\end{gathered}
$$

in which $r=$ radial coordinate; $R_{b}=$ beam radius; $\mathrm{t}=$ time; $T=$ absolute temperature; $T_{0}=$ uniform (hot) temperature established at or near $t=0$ in region $1 ; \underline{v}=$ vector velocity; $v_{r}=$ radial velocity component; a = speed of sound in region 1 at temperature $T_{0}\left(\alpha=\left(\gamma R T_{0} / M\right)^{.5}\right) ; \rho=$ density; $\rho_{0}=$ initial density; $p=$ pressure; $p_{0}=$ pressure at $\rho=\rho_{0}, T=T_{0} ; E=$ internal energy per unit mass; $\omega=$ mass fraction reactant; $w_{0}=$ initial mass fraction reactant; and $k=$ first-order unimolecular rate constant $=A \exp \left(-E_{a} / R T\right)$.

A symmetry boundary condition is applied at the beam axis $(\eta=0)$, which is a singular point of the equations when cast in cylindrical coordinates. A solid wall boundary condition is applied at the reactor wall $\left(\eta=\eta_{r x t r}=R_{r x t r} / R_{b}\right)$. For the ideal case, in which the temperature is uniform throughout the heated region and is established instantaneously, the initial conditions are as follows:

$$
\begin{gathered}
\left\{\varphi=1,0<\eta<\eta_{r x t r}\right\} ; \quad\left\{\underline{\nu}=\underline{0}, 0<\eta<\eta_{r x t r}\right\} ; \quad\left\{\omega=1,0<\eta<\eta_{r x t r}\right\} ; \\
\left\{\Theta=1,0<\eta<1, \Theta=\Theta_{\mathrm{S}}, 1<\eta<\eta_{r x t r}\right\} ; \\
\left\{\pi=1,0<\eta<1, \pi=\Theta_{s}, 1<\eta<\eta_{r x t r}\right\} ; \\
\left\{\xi=1 /(\gamma-1), 0<\eta<1, \xi=\Theta_{s} /(\gamma-1), 1<\eta<\eta_{r x t r}\right\}
\end{gathered}
$$

Examination of the equations and initial conditions presented above show that in this idealized situation the gas dynamics depend on only two dimensionless parameters, which are $\mathrm{y}$, the specific heat ratio, and $\Theta_{s}$, the ratio of the temperature outside the heated region to the temperature in the heated region. Furthermore, these two parameters take on only a small range of values. $\Theta_{s}$ varies from about 0.2 to 0.5 and $\gamma$ varies from about 1.1 to 1.3 for practical experimental conditions. The species balance depends on two additional parameters, the dimensionless preexponential factor and activation energy for the reaction. These take on a wider, but still limited, range of values. 
The above equations and conditions make several assumptions which will be briefly validated. First, conduction, diffusion, and viscous forces are neglected. These effects simply are not important on the time scale of a few microseconds during which reaction takes place. Rough estimates indicate that these processes become important on a time scale of tens of milliseconds. Second, effects of chemical reaction (change in total number of moles, energy production/consumption) are neglected. This is justified by the facts that (a) the reactant is generally present in low concentration in a bath gas and (b) we are considering the case of low per pulse reactant conversion (< $1 \%$ conversion). Consideration of these effects would unnecessarily complicate the analysis. Third, a constant specific heat is assumed, primarily to simplify the calculations. Some calculations were carried out using a specific heat that varied linearly with temperature, and the change in the calculated amount of reaction which occurred was negligible compared to the use of a constant specific heat equal to that at the initial hot temperature. Finally, the equations contain the implicit assumption that thermal equilibrium is attained locally so that the use of an equation of state makes sense. This will be valid when the dimensions of the beam and reactor are very large relative to the mean free path of the gas molecules, which is the case for all practical operating pressures and reactor dimensions.

The above nonlinear hyperbolic partial differential equations with their associated initial and boundary conditions were integrated numerically to obtain the results presented below. Two finite difference based techniques were used. In a few cases, a simple artificial viscosity method utilizing the Lax-Friedrichs numerical flux was applied [11]. This method yielded first-order accuracy and required the use of very small time steps in the integration. Most of the results were obtained using the flux based essentially nonoscillatory (ENO) methods for hyperbolic PDEs described by Shu et al. $[12,13]$. A second-order ENO flux was used with third order Runge-Kutta time stepping. This method yielded sharper shock fronts and allowed the use of larger time steps than the simpler Lax-Friedrichs based method. These techniques are appropriate for the situation considered here in which nonlinear effects are important and discontinuous solutions are expected. Jacobs [14]. has presented a numerical solution method for the problem arising from this same physical situation for the case where the temperature changes and velocities are small so that nonlinear effects are negligible and smooth solutions are expected. The pseudospectral method used by Jacobs is not applicable when the solutions are not smooth, but allows the inclusion of conduction, diffusion, and viscous forces which will be important for the situation considered in [14].

A typical solution $\left(\gamma=1.2, \Theta_{s}=0.3, \eta_{r x t r}=2\right)$ is presented in Figures 2 and 3 . The initial dimensionless profiles are shown in Figure 2(a). In Figure 2(b), corresponding to a dimensionless time of $\tau=0.5$, the head of the expansion wave has reached $\eta=0.5$, the tail is slightly behind that location, the shock has reached about $\eta=1.4$, and the boundary between the hot and cold zones has moved almost to $\eta=1.2$. If we compare this to Figure 1 , we see that $0<\eta<0.5$ corresponds to region 1 , $0.5<\eta<0.75$ corresponds to region $2,0.75<\eta<1.2$ corresponds to region $3,1.2<$ $\eta<1.4$ corresponds to region 4 , and $1.4<\eta<2.0$ corresponds to region 5 . Figure 2(c, d) shows the profiles at dimensionless times of $\tau=0.75$ and $\tau=1$. We see that the front of the expansion wave reaches the axis at $\tau=1$, as it should. At later times, the waves reflect from the axis and wall, and things become a bit more complicated. Figure 3 shows dimensionless temperature as a function of time at four radial positions. We see from this picture that the temperature drops off sharply 


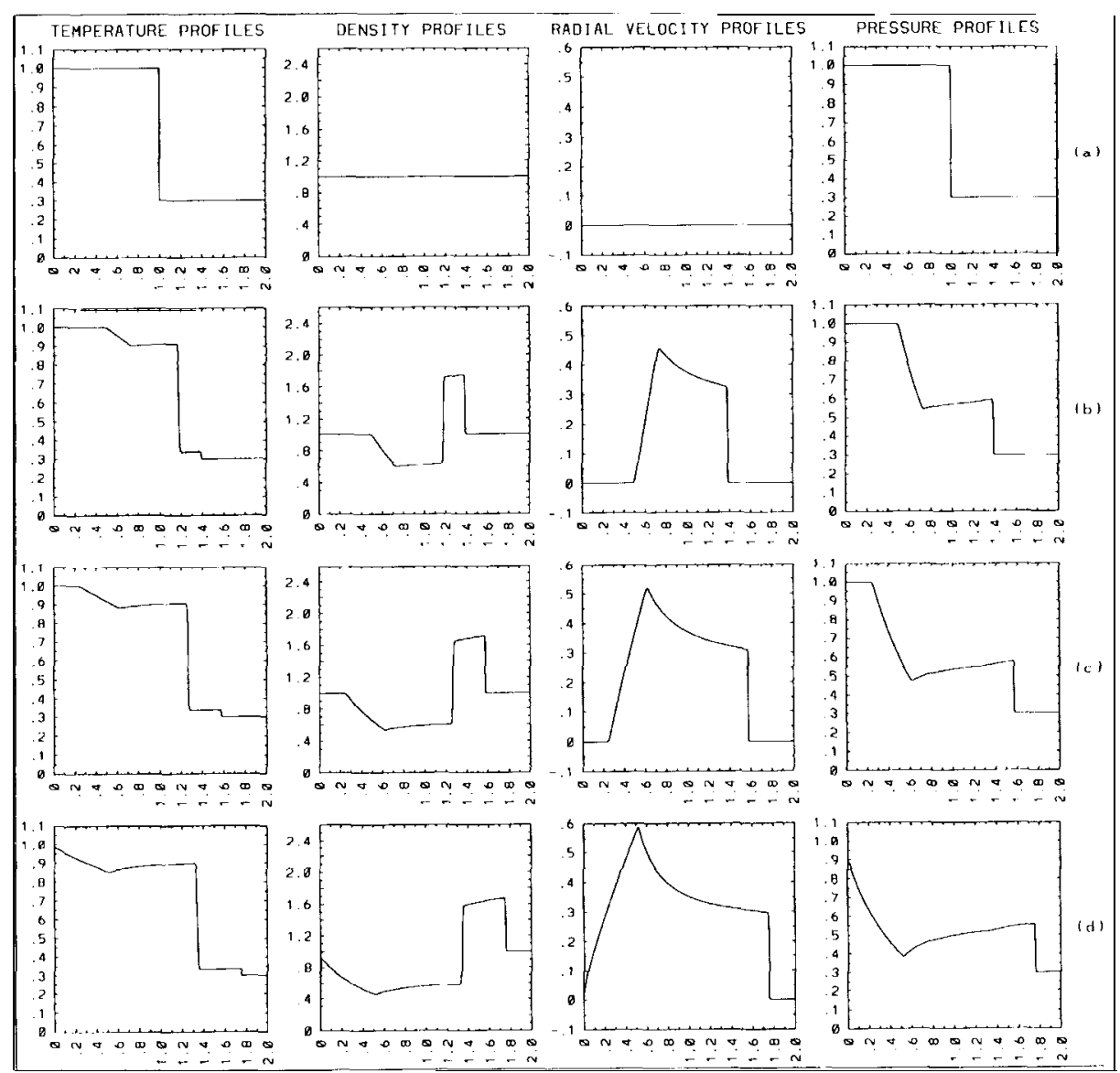

Figure 2. Typical numerical solution of the gas dynamics equations. Parameter values are $\gamma=1.2$ and $\Theta_{s}=0.3$. Spatial profiles of dimensionless temperature, density, radial velocity, and pressure are shown. (a) Initial conditions $(\tau=0)$, (b) $\tau=0.5$, (c) $\tau=0.75$, and $(\mathrm{d}) \boldsymbol{\tau}=1.00$.

after the passage of the head of the expansion wave, and never rebounds to the initial temperature. This leads to the conclusion that reaction is essentially over after the expansion reaches the center at $\tau=1$, and we need not worry much about what happens at later times. This is only partially true, since if the beam and reactor are concentric, the shock wave can reflect from the reactor wall and reheat the center of the reactor to temperatures even higher than the initial temperature. This effect is easily avoided by using a toroidal cell and/or positioning the laser beam off the cell axis so that reflected shocks do not interfere constructively $[7,9]$.

\section{Results and Discussion}

\section{Inherent Error Due to Reaction Behind Expansion Front}

One source of error in the elementary analysis presented above is the assumption that reaction takes place entirely in front of the expansion wave (region 1). Clearly 


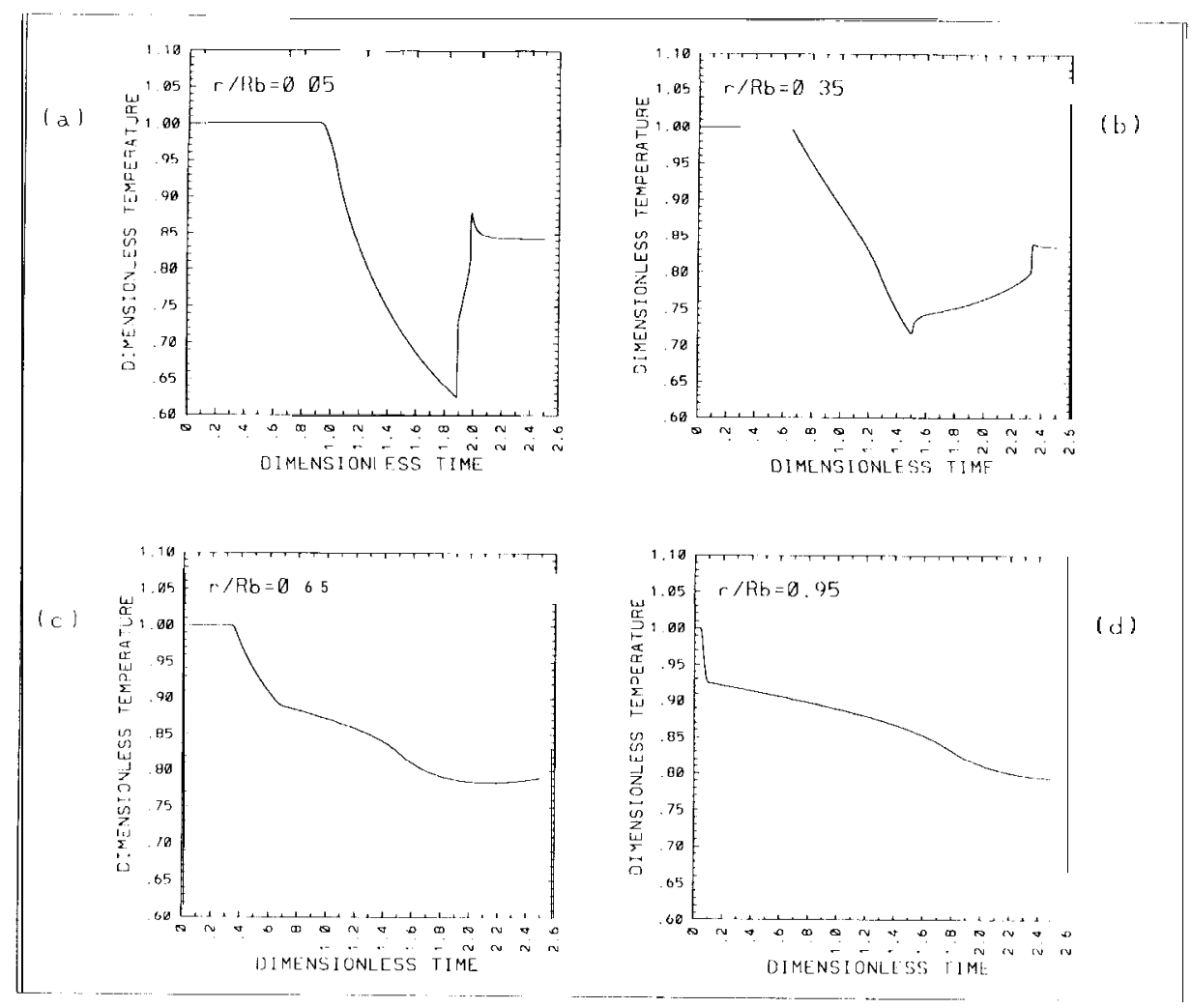

Figure 3. Typical numerical solution of the gas dynamics equations. Parameter values are $\gamma=1.2$ and $\Theta_{s}=0.3$. Temporal profiles of the dimensionless temperature are shown for four radial positions. (a) $\eta=0.05$, (b) $\eta=0.35$, (c) $\eta=0.65$, and (d) $\eta=0.95$.

some reaction will always take place in the region between the head and tail of the expansion wave (region 2). The fraction of chemical reaction which takes place outside of region 1 will depend on how much cooling takes place behind the expansion front, which in turn depends on the values of $\gamma$ and $\Theta_{s}$. It will also depend on the dimensionless activation energy, since it is the Arrhenius dependence of the reaction rate on temperature which serves to quench the reaction. The total amount of reaction which occurs will also depend directly on the preexponential factor, but the fraction occurring in region 1 will be very nearly independent of the preexponential factor, since increasing the preexponential factor simply increases the reaction rate by the same factor everywhere (provided that the per-pulse conversion is small, so that the total concentration is nearly the same everywhere). Calculations were carried out to a time of $\tau=2.5$ to ensure that no further reaction was occurring. Changing this final calculation time had negligible effect on the amount of reaction which occurred, since reaction is essentially complete just after $\tau=1.0$. Plots of the fraction of the total reaction taking place in region 1 as a function of activation energy for various values of $\gamma$ and $\Theta_{s}$ are shown in Figure 4. The four curves on each plot correspond to $\Theta_{s}=0.2,0.3,0.4$, and 0.5 . The fraction of reaction occurring in region 1 decreases with increasing $\Theta_{s}$, since the amount of cooling behind the expansion front decreases with increasing $\Theta_{s}$. Comparing the three graphs shows that the fraction 
of reaction occurring in region 1 increases with increasing $\gamma$, which corresponds to decreasing mixture specific heat. Also, as expected, the fraction of reaction occurring in region 1 increases with increasing dimensionless activation energy. Examination of Figure 4 shows that in order to have most of the reaction occurring in region 1 , where there is a constant, well-defined temperature, the reaction must have a fairly high activation energy, reaction must occur at a high temperature (relative to the ambient temperature), and the gas mixture must have a low specific heat.

The curves shown on the graphs in Figure 4 were generated from a correlation to the simulation results which are shown as points. The correlation reproduces the simulation results to within an average of about $\pm 1 \%$. The correlation is as follows:

$$
\text { fraction }=\left(1.109-0.2800,+\left(1.861-8.231 \Theta_{s}+24.97 \Theta_{s}^{2}\right)\left(\frac{(\gamma+1)}{(\gamma-1)}\right)^{2}\left(\frac{1}{\xi_{a}}\right)^{2}\right)^{-1}
$$

This correlation may also be viewed as a correction factor to be applied to the measured rate constant. Since, in the simple analysis with only the first term of the Taylor series retained, the rate constant is linear in the per pulse conversion, the ratio of the actual rate constant to the rate constant calculated from the measured conversion via the simple analysis is the same as the fraction of reaction occurring in region 1. Thus, rate constants calculated using the simple analysis may be corrected in a straightforward manner to account for reaction taking place behind the expansion front. Since the activation energy must be known in order to apply the correction, this would have to be an iterative process, but very few iterations would be required. It is worth reemphasizing that this correction is inherent in the technique, and cannot be eliminated by improved experimental procedures. This effect limits the range of reactions which may be studied by this technique, since a high activation energy is required. It also places a lower limit on the temperatures which may be used for a given reaction. However, since only the ratio of the reaction temperature to room temperature matters, lower reaction temperatures could be used if the entire reaction cell were cooled. Finally, this effect limits the technique to low specific heat gas mixtures, requiring that a diatomic or small polyatomic bath gas be used in the experiment.

\section{Effect of Axial Temperature Nonuniformity}

Since some of the laser energy is absorbed as it passes through the reaction cell, it is inevitable that there will be a temperature gradient in the cell from the front window to the back window. The magnitude of this gradient will depend on the fraction of the laser energy which is absorbed. The fraction of the laser energy which is absorbed in turn sets the laser power required to heat a given amount of gas to a given temperature. The magnitude of the axial gradient can be reduced substantially by replacing the rear window of the reaction cell with a mirror, so that the IR beam is reflected back through the cell $[1,2,7]$. but some axial temperature gradient will still be present. Clearly this can cause large variations in the reaction rate at different axial positions in the cell. In order to determine how an axial temperature gradient effects the gas dynamics in the reaction cell, some two dimensional calculations were carried out. The resulting temperature profiles are shown in Figure 5. Initially, the temperature is uniform at $\Theta=0.3$ outside the beam radius, and decreases from $\Theta=1.0$ at the front of the cell to $\Theta=0.857$ at the rear of the cell inside the beam. At 

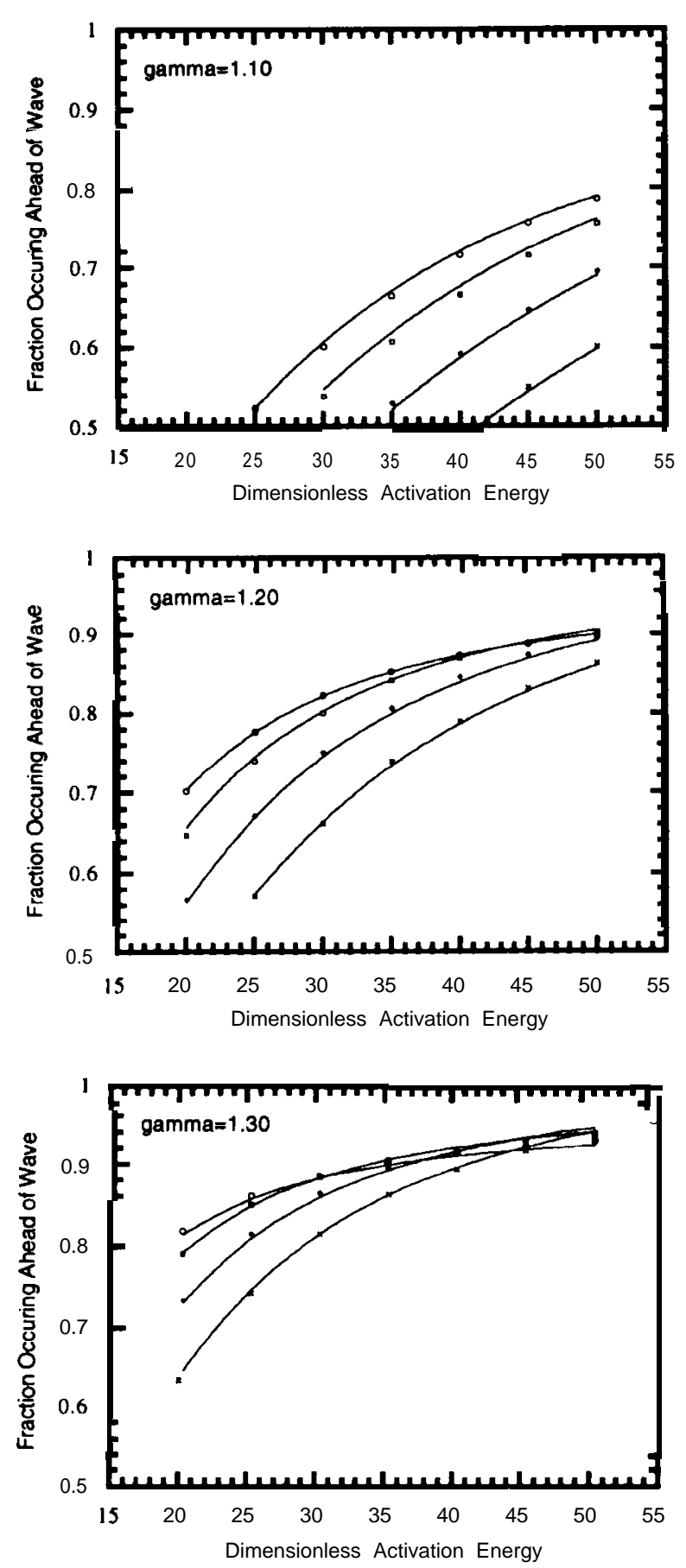

Figure 4. Fraction of reaction which takes place in the uniform temperature region (region 1 in Fig. 1). Points are results of numerical solution of the governing equations. Lines are a correlation to the points (and others not presented), as given in the text. The four curves on each plot correspond to $\Theta_{s}=0.2,0.3,0.4$, and 0.5 , with the lowest curve corresponding to $\boldsymbol{\Theta}_{s}=0.5$. The three graphs are for heat capacity ratios of $\gamma=1.10$, 1.20 , and 1.30 as labelled. 
$\tau=0.5, \tau=0.75$, and $\tau=1.0$, the waves are propagating slightly faster at the front of the cell than at the rear, but overall the axial temperature gradient appears to have little effect on the gas dynamics. It should also be noted that the axial temperature gradient tends to persist. That is, although some axial acoustic waves propagate, tending to cool the hot end and heat the cool end, the temperature remains higher in front than in back throughout the time period when reaction is occurring. Note that the case shown corresponds to a much larger temperature gradient than would be acceptable in practice. For a reaction with a dimensionless activation energy of 35 , the initial reaction rate would decrease by a factor of about 340 from the front to the back of the cell for the case shown. Thus, these calculations show that any axial temperature gradient which is acceptable from the point of view of its effect on reaction rates will have a negligible effect on the gas dynamics. As a simple measure of the effect of axial temperature gradients on reaction rates, if the dimensionless temperature at the front of the cell is 1 and the dimensionless temperature at the rear is 6 , then the ratio of the rate at the rear to that at the front is simply:

$$
\frac{r_{\text {rear }}}{r_{\text {front }}}=\exp \left(\frac{-\xi_{a}\left(1-\Theta_{r}\right)}{\Theta_{r}}\right)
$$

Thus, we may set a criterion for how much difference in reaction rate from front to back we will accept, and then (assuming we have a rough idea of $E_{a}$ ) calculate what temperature gradient is acceptable. From this, it is straightforward to estimate the fraction of the laser power which may be absorbed (depending strongly on whether we reflect the beam) and the laser power requirements for a given gas mixture, pressure, and cell length. Note that we still have the problem of determining what temperature to assign the measured rate constant to. That is, what sort of average temperature is measured by the temperature measurement technique used, and is it the appropriate average to associate with a measured rate constant? This question will be addressed further in the following section dealing with radial temperature nonuniformity.

\section{Effect of Radial Temperature Nonuniformity}

The portion of the laser beam which is used to heat the gas mixture in an actual LPHP experiment will not be perfectly uniform. In order to define a beam which is as nearly uniform as possible, the beam may be partially focused and then the center portion selected by passing the beam through one or more stops $[3,6,8,9]$. We can expect that the beam intensity will be slightly lower at the edge than at the center, and that there may be local bumps or rings in the laser intensity profile due to local hot spots in the multi-mode laser output and/or diffraction from the various optics and stops used. In order to simulate this effect, two situations were considered. In the first an initial temperature profile which decreases from $\Theta=1$ at the beam center to $\Theta=\Theta_{e}$ at the beam edge was considered. The resulting profiles for $\Theta_{e}=0.95$, along with the profiles for a uniform initial temperature, are shown in Figure 6. Figure 6(a) shows the initial conditions. Figures 6(b), (c), and (d) show the profiles at $\tau=0.5$, $\tau=0.75$, and $\tau=1.0$, respectively. Note that the initial temperature profile tends to persist as the expansion wave moves toward the center of the cell. The second case considered was one in which oscillations were superimposed on the uniform initial temperature profile, so that the initial profile was given by $\Theta=1+\epsilon \cos (\beta \eta)$. The resulting profiles for $\epsilon=0.02, \beta=40$ are shown in Figure 7 . Again, note that the 

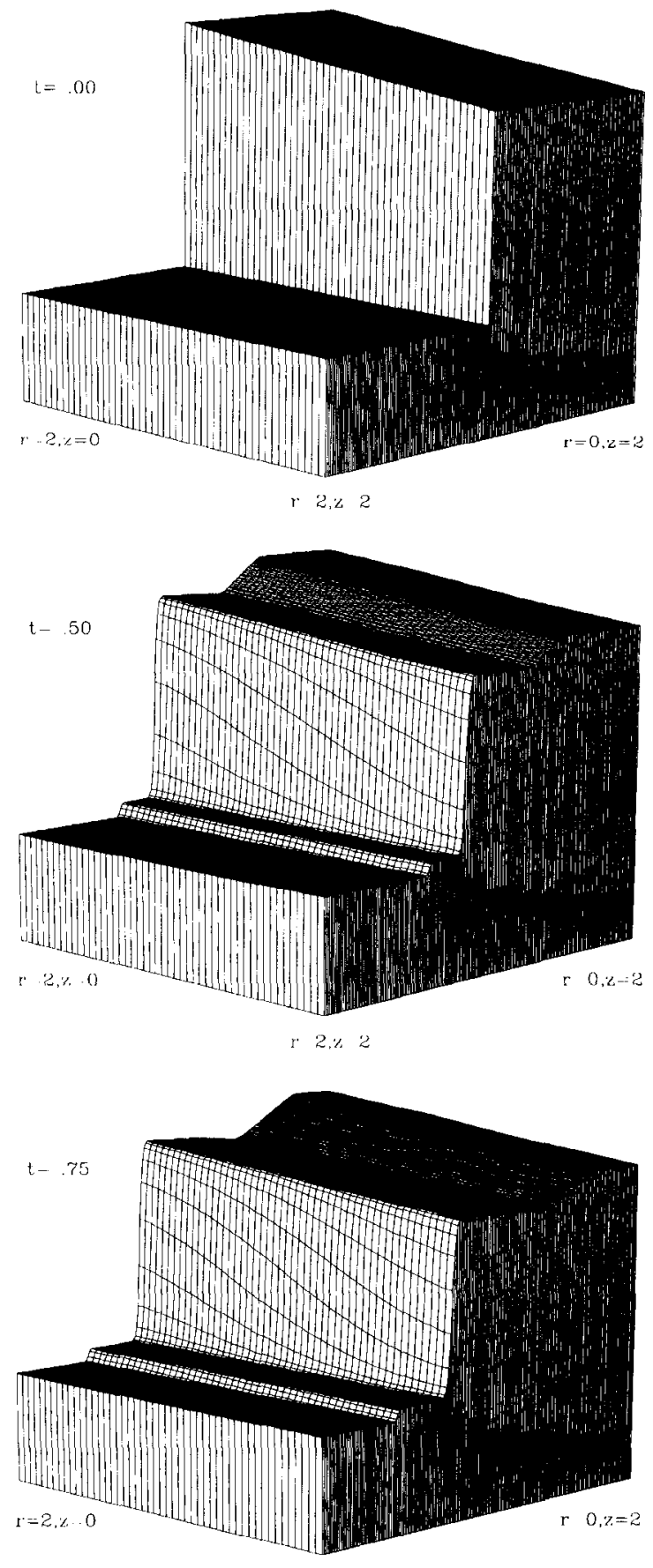

$r \cdot 2 \cdot 7 \cdot-3$

Figure 5. Temperature profiles resulting from two-dimensional calculations. Initially, the temperature in the cold region is $\Theta=0.3$ (outside the beam), and the temperature in the hot region varies from $\Theta=1.00$ to $\Theta=0.857$. The profiles shown are for $\gamma=1.2$. 
which reaction takes place. Also note that the initial oscillations in temperature lead to significant oscillations in the density profile near the center of the beam.

The typical magnitude of the effect of having lower laser energy near the beam edge is shown in Figure 8. In this figure, the relative reactant conversion is referenced to the conversion obtained if the initial dimensionless temperature were uniform at $\Theta=1$, so the relative conversions shown are less than 1 . If the conversion were referenced to the temperature at the beam edge, then the effect would be reversed, and the relative conversions would be greater than 1 . The points shown on the graph are for a single set of conditions with $\gamma=1.2$ and $\Theta_{s}=0.3$, but this effect will depend only weakly on those parameters. The effect increases with increasing $\xi_{a}$ as shown in Figure 8. It is clear from these results that for the situation depicted in Figure 6, where the temperature at the edge of the beam is 5\% lower than that at the center, there may be only half as much reaction as if the temperature were uniform at the center temperature (but twice as much as if it were uniform at the edge temperature). It therefore appears that this could be a source of large errors in the measurement of rate constants by the LPHP technique. However, as with the case of axial temperature nonuniformity, the problem lies primarily in determining the correct temperature to assign the measured rate constant to. This leads directly to the problem of temperature measurement in the LPHP experiment. The effect of temperature nonuniformities on two temperature measurement techniques will be discussed below.

If chemical thermometry is used to measure the temperature, then the correct average temperature will be measured if the activation energy for the decomposition of the thermal monitor molecule is the same as the activation energy for the reaction being investigated $[3,4,7]$. It is of course also necessary that the two reactions are totally independent, a fact which can be difficult to verify. If the activation energies are much different, then different average temperatures will apply to the two reactions, since the effect of temperature variation will be different for the two reactions. Examination of Figure 8 shows that the relative conversion depends weakly on temperature, and that even when the activation energies differ substantially, the relative conversion changes by only a few percent. So we expect chemical thermometry to provide very nearly the correct average temperature for the system, even when the initial temperature profile is not entirely uniform.

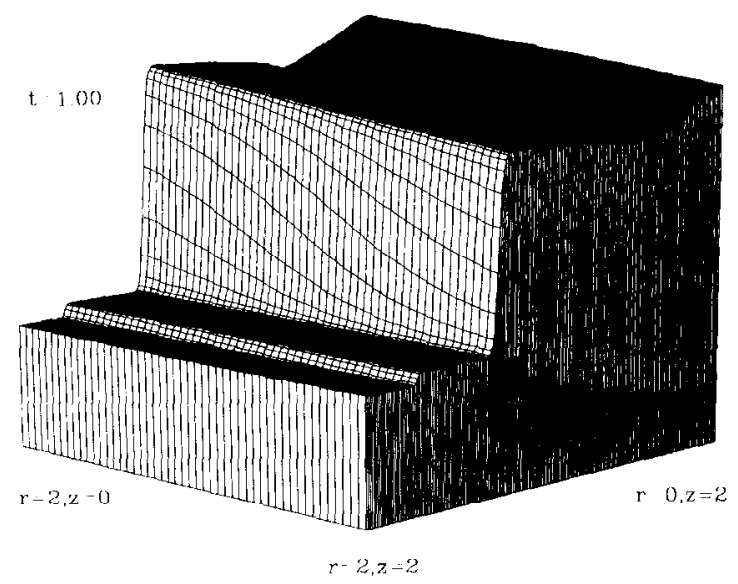

Figure 5. (continued) 


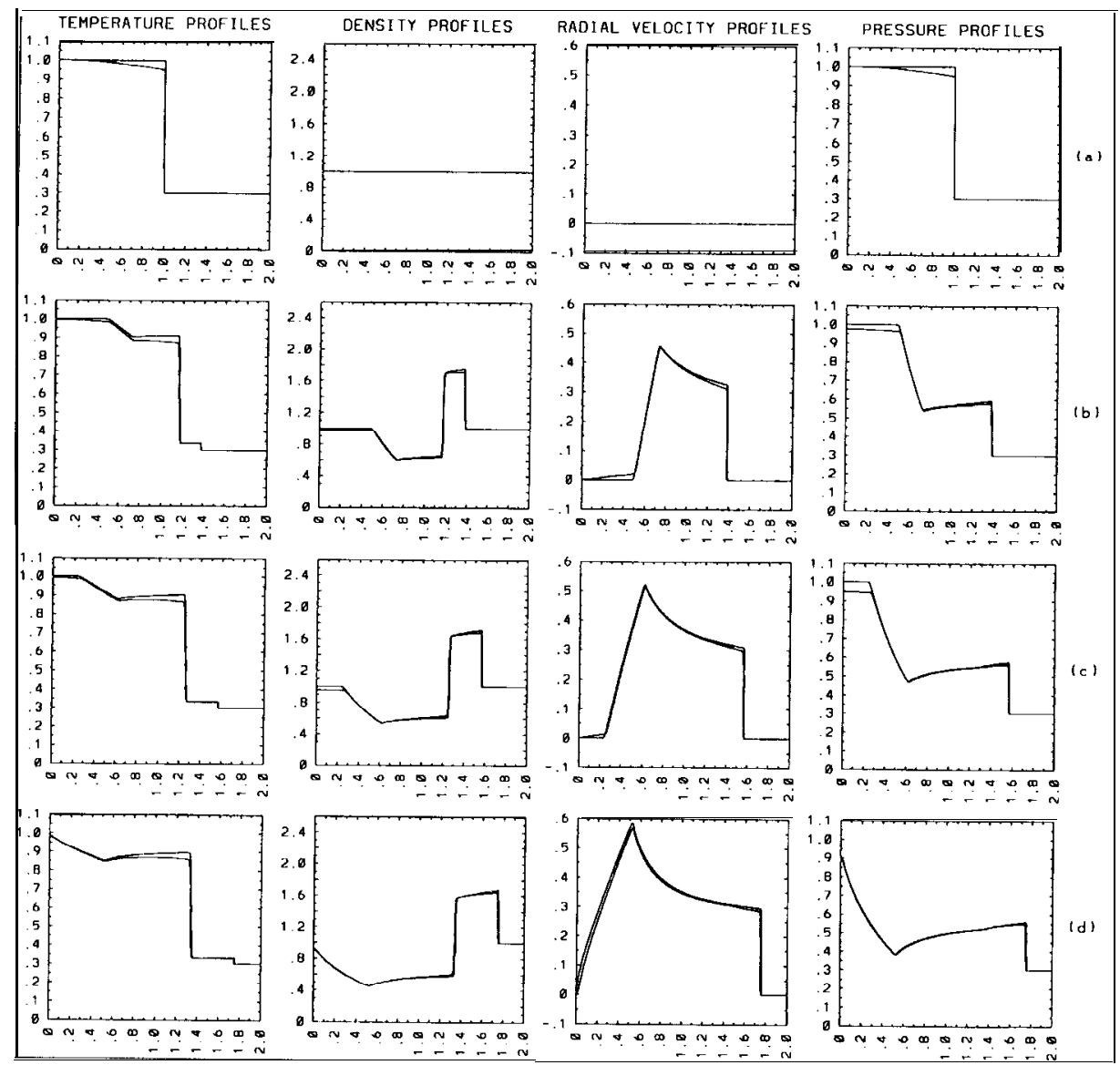

Figure 6. A comparison of the profiles resulting from an initially uniform temperature and a temperature distribution which drops to $\Theta_{e}=0.95$ at the beam edge. Parameter values are $\gamma=1.2$ and $\Theta_{s}=0.3$. Spatial profiles of dimensionless temperature, density, radial velocity, and pressure are shown. (a) Initial conditions $(\tau=0),(b) \tau=0.5$, (c) $\tau=0.75$, and (d) $\tau=1.00$.

In many cases it may be difficult or impossible to find a well-characterized thermal monitor molecule which reacts independently of the reactant being studied. In these cases, time dependent thermal lens measurements may be used to determine the temperature of the system [8,9]. In this method, a second smaller laser beam (usually a HeNe beam) is combined coaxially with the IR beam. This second beam is refracted by the gas in the cell and focused or defocused by density gradients in the gas, since the index of refraction of a gas is density dependent $[8,15,16]$. For gases at pressures less than atmospheric pressure, the index of refraction is approximately given by $n=1.5^{*}(A / M) \rho$, where $\boldsymbol{A}$ is the molar refractivity of the gas [15]. The energy of the transmitted beam (selected through a pinhole) is monitored, and the temperature history of the gas can be inferred from these measurements. The thermal lens signal is defined as $\mathrm{S}=1-I / I_{0}$, where $I$ is the measured intensity and $I_{0}$ is the intensity of the unperturbed beam. For small signals, $S$ is proportional to the curvature of the gas density profile on the beam axis (at $\eta=0$ ) [15]. In the LPHP experiment, a sharp, negative spike in this thermal lens signal occurs when the head 


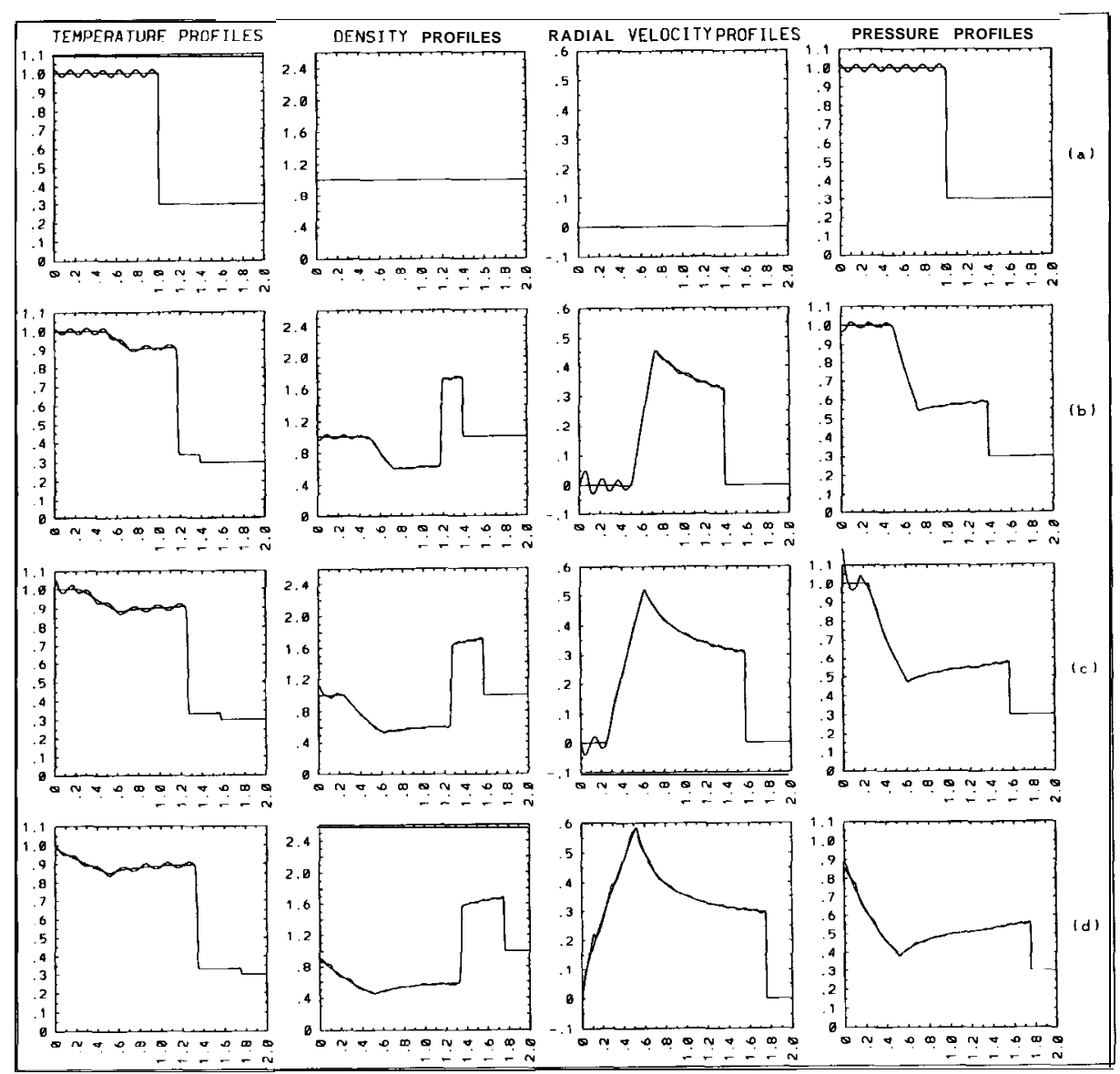

Figure 7. A comparison of the profiles resulting from an initially uniform temperature and a temperature distribution with oscillations superimposed. The amplitude of the oscillations is $2 \%$ of the absolute temperature. Parameter values are $\gamma=1.2$ and $\Theta_{s}=0.3$. Spatial profiles of dimensionless temperature, density, radial velocity, and pressure are shown. (a) Initial conditions $(\tau=0)$, (b) $\tau=0.5$, (c) $\tau=0.75$, and (d) $\tau=1.00$.

of the expansion wave reaches the axis of the beam. From the delay between the laser firing and the spike, combined with knowledge of the initial beam diameter, we may determine the (temperature dependent) speed of sound in the gas, and hence the temperature [8]. If the initial temperature profile is nonuniform, then the delay time will reflect some average speed of sound in the hot region, which will lead to the measurement of some average temperature. Simulated thermal lens signals were generated by solving the gas dynamics equations numerically and computing the second derivative of the density near $\eta=0$. These are shown in Figure 9. In Figure 9(a) the thermal lens signals are obtained starting with an initially uniform temperature profile and then with a profile that drops to $\Theta_{e}=0.95$ at the beam edge. The only significant difference between these profiles is that the delay time between the laser firing and the spike is slightly longer when the edge temperature is lowered. From observation of several simulated thermal lens signals such as this one, it appears that the delay time corresponds very closely to the temperature 


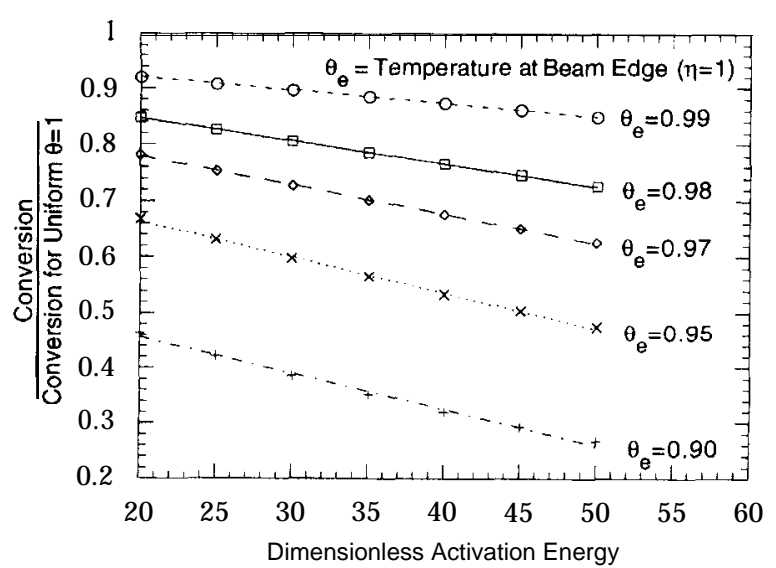

Figure 8. Typical effect on total per pulse conversion of an initial temperature profile which drops to $\Theta=\Theta_{e}$ at the beam edge. Values shown are the calculated conversion divided by the calculated conversion if the temperature were uniform. Parameters are $\gamma=1.2$ and $\Theta_{s}=0.3$.

at the edge of the beam. Thus, measurement of the temperature via thermal lens techniques will tend to yield the temperature at the edge of the beam, which may be lower than the appropriate average temperature for the reaction. Figure 9(b) shows simulated thermal lens signals for the initially uniform temperature profile and for the temperature profile with oscillations superimposed $(\epsilon=0.005, \beta=40)$. Small bumps in the initial temperature profile $(0.5 \%$ of the absolute temperature) lead to large oscillations in the thermal lens signal. However, the large negative spike which is used in measuring the temperature is still clearly visible and occurs at the same

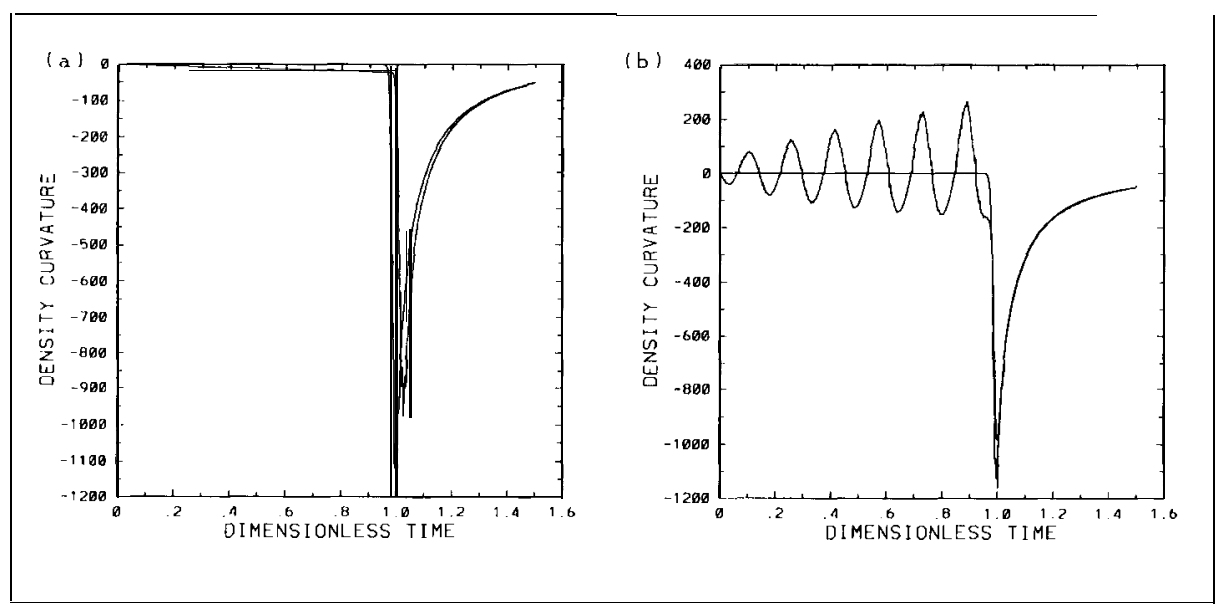

Figure 9. Simulated thermal lens signals, plotted as the second derivative of the density profile near $\boldsymbol{\eta}=0$ vs. dimensionless time. Parameters are again $\gamma=1.2$ and $\Theta_{s}=0.3$. (a) Signals resulting from an initially uniform temperature profile and from an initial temperature profile which drops to $\Theta_{e}=0.95$ at the beam edge. (b) Signals resulting from an initially uniform temperature profile and from an initial temperature profile with oscillations (of amplitude equal to $0.5 \%$ of the absolute temperature) superimposed. 
time as for the uniform initial temperature profile. Thus, the existence of oscillations in the thermal lens signal is not necessarily indicative of large fluctuations in the initial temperature profile, and we should not expect to measure thermal lens signals which are initially flat, as would be expected with a perfectly uniform initial temperature profile.

Radial temperature nonuniformity has been shown to have a large effect on measured rate constants, but, as with axial temperature nonuniformity, the problem is largely one of determining the appropriate temperature to associate with the kinetic measurements. Chemical thermometry, when applicable, is expected to do a good job of measuring the appropriate temperature. Thermal lensing has been found to measure the temperature at the beam edge, which may be lower than the appropriate average. Other temperature measurement techniques may measure different average temperatures. In any case, the ratio of the initial rate at the center of the beam to that at the edge may be calculated from:

$$
\frac{r_{\text {edge }}}{r_{\text {center }}}=\exp \left(\frac{-\xi_{a}\left(1-\Theta_{e}\right)}{\Theta_{e}}\right)
$$

where $\Theta_{e}$ is the dimensionless temperature at the beam edge. As in the case of axial temperature variation, this expression may be used as a guideline for calculating the acceptable variation in laser intensity (and hence temperature), or to estimate the effect on reaction rates of a known radial intensity profile. Clearly, the use of more than one temperature measurement technique in a given experiment should be beneficial. For example, agreement between temperatures measured via chemical thermometry and by thermal lensing serves both to indicate that the monitor reaction and reaction of interest are independent and to confirm that there was a reasonably uniform initial temperature distribution.

\section{Effect of Noninstantaneous Energy Deposition}

All of the calculations and analysis presented thus far have made the assumption that the initial temperature profile is established instantaneously. This implies both

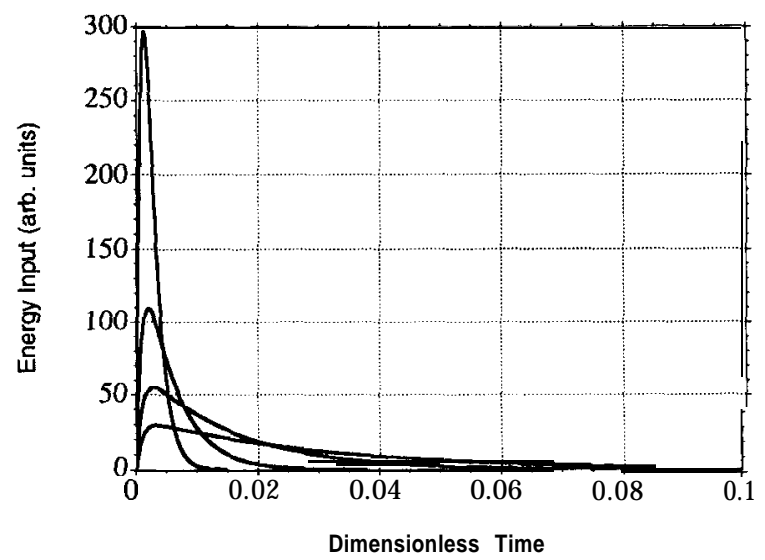

Figure 10. Energy pulse shapes used as input to calculations with noninstantaneous energy deposition. These are of the form $S_{E}(\tau)=\mathrm{C}\left(\exp \left(-\tau / \tau_{1}\right)-\exp \left(-\tau / \tau_{2}\right)\right)$, scaled to give the desired final temperature. The four pulses shown all have $\tau_{2}=0.001$, and have $\tau_{1}=0.0015,0.005,0.015$, and 0.03 . 
that the laser pulse is instantaneous and that the energy transfer from vibrational modes of the sensitizing molecule to all of the modes of all of the molecules is instantaneous. In reality, the laser pulse may have a duration from $100 \mathrm{~ns}$ to more than $1 \mu \mathrm{s}$, depending on the laser configuration and the gas mixture used in the laser. Energy transfer rates are not easily characterized, but can be made fast by increasing the pressure in the reactor. Increasing the pressure, however, increases the total heat capacity of the system, requiring more laser power to heat a fixed volume of gas to a given temperature. In order to examine the effect of noninstantaneous energy deposition into the gas, calculations were carried out in which the reactor contents were initially all at the same temperature, and then energy was added to the system in a short pulse to establish the final temperature. The pulse shapes used are shown in Figure 10. In this situation, some heating occurs nearly instantaneously, but the final temperature is not established until the pulse is nearly complete. Since the speed of propagation of the expansion wave is only weakly temperature dependent, it starts moving at nearly its final velocity almost immediately. However, since the reaction rate is strongly temperature dependent, very little reaction occurs until the final temperature is reached. Thus, the expansion wave has already moved before significant reaction occurs, and both the effective reaction volume and the effective reaction time are shorter than if the temperature profile were established instantaneously. Figure 11 shows the effect of the four pulses shown in Figure 10 on the conversion of reactant for a typical case $\left(\gamma=1.2, \Theta_{s}=0.3\right)$. The magnitude of the effect depends only weakly on the other experimental parameters, increasing slightly with increasing $\gamma$, and decreasing slightly with increasing $\Theta_{s}$. Clearly, this is a small effect for cases where all of the energy is deposited in less than about one twentieth of the time required for the expansion wave to transverse the beam radius. Knowing this delay time for a given reaction temperature and beam diameter, we therefore have a criterion for the acceptable duration of the laser pulse. We also require that energy transfer from the sensitizer molecule to the reactant and vibrational-translational energy transfer take place on the same time scale. This may be accomplished by increasing the operating pressure of the system, based on whatever energy transfer data are available for the gas mixture being considered.

\section{Effects of Pulse-to-Pulse Variations in Initial Temperature}

The energy content of the $\mathrm{CO}_{2}$ laser pulse is not perfectly reproducible from pulse to pulse. Thus, in a typical experiment where 100 laser pulses are fired, and a rate constant is determined from the final conversion, some pulses will have contributed more than others. Due to the Arrhenius temperature dependence of the rate constant, pulses in which the temperature is higher than average will overcontribute much more than pulses in which the temperature is below average will undercontribute. This means that the measured rate constant will be higher than the actual rate constant at the temperature associated with the mean laser pulse energy. In order to investigate the magnitude of this effect, Monte-Carlo type simulations were carried out, in which initial dimensionless temperatures were selected from a normal distribution with a mean of 1 and a standard deviation of 0.01 to 0.1 . The conversion after 100 (or some other fixed number) of pulses was computed, then a measured rate constant was extracted from that conversion. The whole process was repeated 10,000 or so times to yield an average overestimate of the rate constant due to these pulse-to-pulse variations in temperature. The results are presented in Figure 12 as the ratio of the 


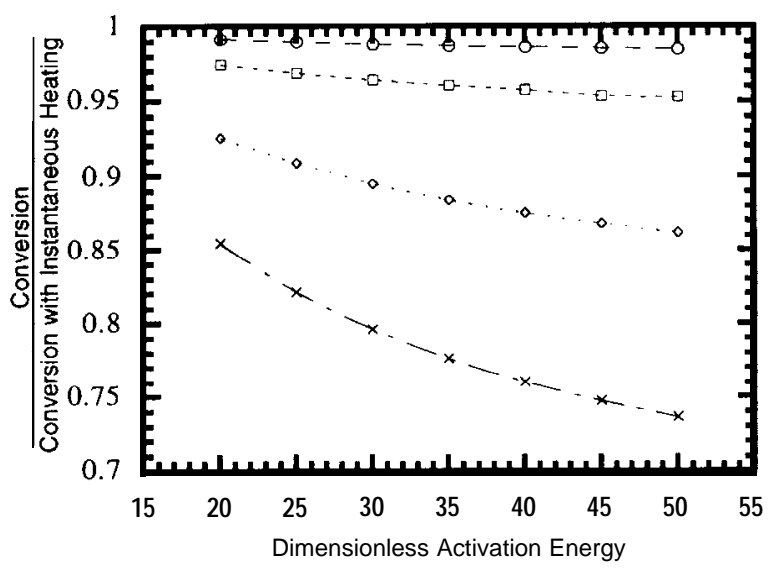

Figure 11. Typical result of noninstantaneous energy deposition. The four curves shown correspond to the four pulses shown in Figure 10. Values shown are the ratio of the per pulse conversion divided by the total conversion if the final temperature were attained instantaneously. Parameter values are $\gamma=1.2$ and $\Theta_{s}=0.3$.

average measured rate constant to the actual rate constant at the mean temperature, plotted vs. the standard deviation of the dimensionless temperature distribution for various activation energies. These are typical results and will vary slightly with the reactor volume, $\gamma, \Theta_{s}$, number of pulses per experiment, and the preexponential factor. From Figure 12, we see that it is necessary to have pulse-to-pulse temperature reproducibility with a standard deviation of less than 0.02 . This means that about $2 / 3$ of the laser pulses need to be within $2-3 \%$ of the mean energy, which is not a very stringent requirement to impose on the laser. Thus, if the laser pulses are

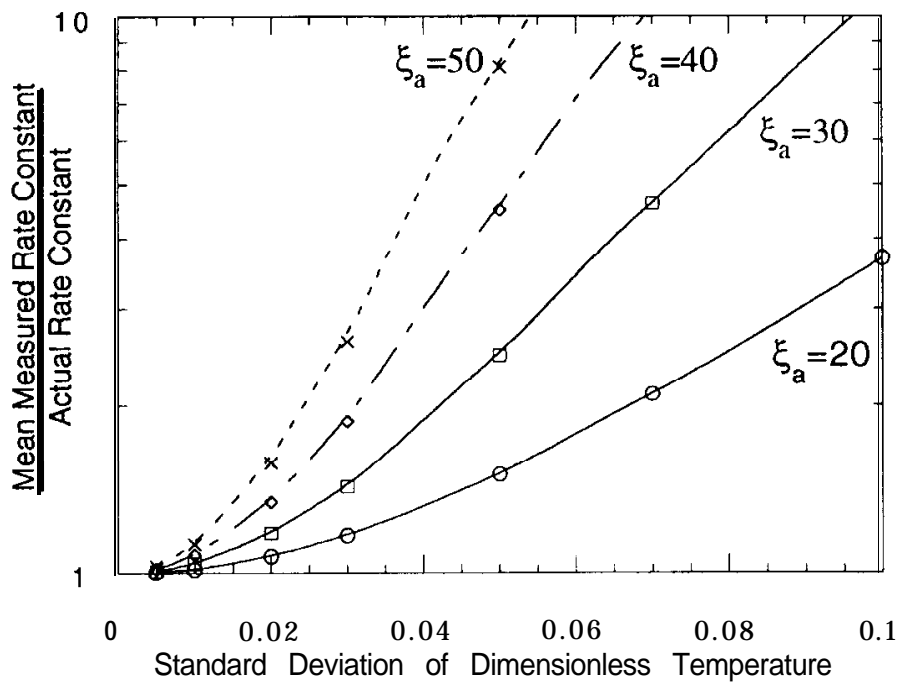

Figure 12. Typical result of pulse-to-pulse variations in the initial temperature profile. Results shown are for 100 pulses per experiment and a reactor in which $4 \%$ of the reactor volume is initially heated. The preexponential factor has been adjusted to give a dimensionless rate constant of $\boldsymbol{\kappa}=0.01$ for each activation energy. The four curves shown correspond to dimensionless activation energies of $\xi_{\alpha}=20,30,40$, and 50 . 
reasonably reproducible, this effect will not be important. However, it is necessary to carry out experiments with a large number of pulses $(>100)$ per experiment to reduce the effect of the occasional unusually high energy pulse. Clearly, some random error is introduced into the experiment by imperfect pulse-to-pulse reproducibility, and this error is minimized when a large number of pulses are used, either by using many pulses per experiment, or by repeating the experiment many times and carrying out the appropriate averaging.

\section{Conclusions}

The calculations presented in this article provide a basis for design of pulsed LPHP kinetics experiments, and for estimation of the accuracy with which rate constants may be determined by this technique. A correlation was presented which may be used to correct for the inherent error of the simple analysis due to reaction occurring outside of the uniform temperature region. These results also pointed out that in order for most of the reaction to occur in the uniform temperature region, an appropriate combination of a low specific heat bath gas, a high reaction temperature, and a high reaction activation energy are required. Axial temperature uniformity, determined by the fraction of laser energy absorbed, and radial temperature uniformity, determined by the laser intensity profile, were shown to have large effects on the measured rate constants. These effects, however, were shown to be largely a matter of determining the appropriate average temperature to associate with a given measured rate constant. Chemical thermometry, when applicable, should give very nearly the best average temperature. Thermal lens measurements will tend to give the temperature at the edge of the beam. Errors due to noninstantaneous energy deposition were found to be negligible when the ratio of the laser pulse length to the reaction time was less than about 0.05 . Errors due to pulse-to-pulse variations in laser intensity were found to be negligible when the laser pulses were reproducible within about $2 \%$ and a large number of pulses per experiment were used. Additional requirements for success of the pulsed LPHP experiment are that the reactor pressure be high enough that energy transfer from the sensitizer to the other species is complete in less than about $5 \%$ of the reaction time, and that a toroidal cell geometry and/or off center beam placement are used to avoid reheating of the gas mixture by the reflected shock wave. A complication that has not been treated in this article is the fact that many reactions of interest will be in the pressure dependent fall-off regime at the pressures of less that 100 torr which are most convenient for this experiment. The laser power requirements for this experiment will depend on the desired beam diameter, reaction temperature, heat capacity of the gas mixture, operating pressure, cell length, and other experimental parameters, but it appears that powers on the order of one to a few joules per pulse should be sufficient, with higher powers being preferable. TEA $\mathrm{CO}_{2}$ Lasers providing up to $20 \mathrm{~J} /$ pulse with a $200 \mathrm{~ns}$ pulse duration at $0.5 \mathrm{~Hz}$ are commercially available. The multimode output of this type of laser is essentially uniform, but may have local hot spots. It should also be noted that decomposition of the sensitizer may place a practical limit on the amount of energy that can be supplied to a given reactor volume at a given pressure.

If we consider the effects analyzed here to be the major sources of error in a LPHP experiment, we may obtain a coarse estimate of the overall reliability of the measured rate constant. If there are uncertainties in the chemistry then this will not be the case. In a well designed experiment, the primary source of error considered 
here (after correcting for reaction occurring behind the rarefaction wave> will be uncertainty in the temperature. The amount of error this introduces into the rate constant measurement depends strongly on the activation energy, but if the absolute temperature is uniform to within 5\% and we measure the temperature at some point in the reactor so that the measured temperature is within about $3 \%$ of the correct average temperature, then for a reaction with a dimensionless activation energy of 35 the rate constant will be uncertain by about a factor of 2 . When a high power laser with a uniform beam profile is used and/or a well characterized thermal monitor molecule is available which decomposes with an activation energy close to that of the reactant being investigated we can expect the results to be more precise.

\section{Acknowledgment}

We would like to thank the Minnesota Supercomputer Institute for a grant of time on the Cray-XMP, and the National Science Foundation for support in the form of a graduate fellowship.

\section{Bibliography}

[1] K.E. Lewis, D. M. Golden, and G.P. Smith, J. Am. Chem. Soc., 106, 3905 (1984).

[2] G.P. Smith and R.M. Laine, J. Phys. Chem., 85, 1620 (1981).

[3] H. Dai, E. Specht, M. R. Berman, and C. B. Moore, J. Chem. Phys., 77, 4494 (1982).

[4] C. Steel, V. Starov, R. Leo, P. John, and R. G. Harrison, Chem. Phys. Lett., 62, 121 (1979).

[5] J. Blazejowski, J. Rak, and F. W. Lampe, J. Photochemistry and Photobiology, A: Chemistry, 52, 247 (1990).

[6] D.K. Russell, Chem. Soc. Rev., 19, 407 (1990).

[7] D. F. McMillen, K. E. Lewis, G. P. Smith, and D. M. Golden, J. Phys. Chem., 86, 709 (1982).

[8] J.R. Guckert and R. W. Carr, J. Phys. Chem., 90, 4286 (1986).

[9] P.M. Kuchibhotla, PhD Thesis, University of Minnesota, 1989.

[10] R. B. Bird, W. E. Stewart, and E. N. Lightfoot, Transport Phenomena, Wiley, 1960.

[11] J. C. Strikwerda, Finite Difference Schemes and Partial Differential Equations, Wadsworth and Brooks, 1989.

[12] C. Shu and S. Osher, J. Comput. Phys., 77, 439 (1988).

[13] C. Shu and S. Osher, J. Comput. Phys., 83, 32 (1989).

[14] S. J. Jacobs, Chem. Phys., 132, 71 (1989).

[15] J.R. Barker and T. Rothem, Chem. Phys., 68, 331 (1982).

[16] R.T. Bailey, F.R. Cruikshank, R. Guthrie, D. Pugh, and I. J.M. Weir, Mol. Phys., 48, 81 (1983).

Received November 30, 1993

Accepted January 27, 1994 